\title{
Chemical targeting of voltage sensitive dyes to specific cell types in the brain
}

\author{
Tomas Fialall, Jihang Wang\|, Matthew Dunn, Se Joon Choi, Peter Šebej, Ekeoma C. \\ Nwadibia, Eva Fialova, Diana M. Martinez, David Sulzer* and Dalibor Sames*
}

\section{Slide 1}

Hello. My name is Tomas Fiala. I am a graduate student at the Department of Chemistry, Columbia University in New York in the laboratory of Dalibor Sames. My presentation is going to be about "Chemical Targeting of Voltage Sensitive Dyes to Specific Cell Types in the Brain".

\section{Slide 2}

First, I would like to give you a brief introduction into the world of optical voltage imaging. Recording membrane potential changes in excitable cells has been a challenge for decades. Apart from classical tools like electrophysiology, optical tools have been developed, which include fluorescent molecules which change their absorption or emission properties with changes in membrane potential. One class of these are protein-based sensors, so called GEVIs (or genetically encoded voltage indicators). You see an example of a GEVI design in the figure - a voltage sensitive domain fused with a fluorescent protein. These sensors are very useful, mainly due to the possibility of expressing these in specific cell population by using cell-type specific promoters. However, many problems are also associated with their use including the need for genetic manipulation and need for optimization in every model system. Also, perturbation of the membrane properties is not uncommon. On the other hand, synthetic small molecule voltage sensitive dyes (or VSDs) have been developed. These often exhibit favorable photophysical properties and voltage sensitivity compared to GEVIs and are usable in all species without genetic manipulation. The major drawback of the use of VSDs is their inherent high lipophilicity which makes them sticky to all cell membranes and renders their targeting to specific cell types very challenging, especially in brain tissue. If you are interested in this topic more, I recommend two reviews cited in the lower left corner. In our work, we aimed to fuse the advantages of proteinbased GEVIs and synthetic VSDs and develop a purely chemical platform that would enable delivery of VSDs to specific cell types in brain tissue without genetic manipulation and with the use of ligands for natively expressed proteins. 


\section{Slide 3}

The picture on the top captures the general concept of our targetable voltage sensor. The blue ribbon represents a polymer carrier which carries a voltage sensor (the pink star) and a targeting ligand (the green triangle). In bulk solution, the polymer encapsulates or shields the VSD from the surrounding aqueous environment. The lipophilic VSD then does not bind to cells that do not express the target protein of interest, like the red cell. Only upon binding of the green ligand to the target receptor (which is shown in orange), the voltage sensor is deployed onto the cell of interest.

The specific design of our sensor is shown below. We chose a small, $6 \mathrm{kDa}$ dextran polymer as a carrier, color coded in blue. This is a bacterial polysaccharide with multiple advantageous properties such as biocompatibility but at the same time stability in mammals due to their lack of enzymes for breaking down the non-classical 1-6 alpha glycosidic linkages. As the targeting component, color coded in green, we used dichloropane which is a tropane-class ligand which binds with low nanomolar affinity to the monoamine transporters, that is to the dopamine transporter (or DAT) and norepinephrin transporter (or NET). Finally, the voltage sensor is color coded in purple. We used one of the state-of-the-art photoinduced electron transfer-based sensors developed by the group of Dr. Evan Miller at Berkeley.

\section{Slide 4}

To enable facile attachment of the ligand and the voltage sensor to the dextran polymer, we first developed a functionalization protocol for attaching azidopropyl carbamate linkers to dextran. By reacting dextran with the corresponding activated para-nitrophenyl carbamate, we were able to prepare azido-functionalized dextran with a high degree of control over the number of $\mathrm{N}_{3}$ groups per polymer. Then, we used strain promoted azide alkyne cycloaddition click chemistry to assemble the final probe. We used bicyclononyne-functionalized ligand and dye in one pot, using 0.7 equivalents of VSD and 1.4 equivalents of ligand. This assembly process is to a large degree stochastic, thus we used sub-1-equivalent of dye to ensure that we have a minimum number of dextran molecules in our product which carry 2 or more VSD molecules (which would increase its lipophilicity and presumably non-specific binding). Similarly, we used an excess of ligand to ensure that each dextran molecule holds at least one ligand.

Because our probes contain a voltage sensor, a ligand and a dextran and target monoamine transporters, we termed them VoLDeMo probes.

\section{Slide 5}

We observed a very interesting behavior of VoLDeMo probes by proton NMR spectroscopy. The NMR spectrum on top represents the aromatic region of the spectrum of dsRVF5-VoLDeMo in 
D6-DMSO, an aprotic organic solvent. All signals, both of the VSD and ligand, are well resolved, indicating a high degree of conformational freedom of these components. A contrasting picture, however, is seen in the spectrum recorded in deuterated water, the bottom one. Only three sharp signals remain (marked with green stars), while the remaining signals are highly broadened. The three sharp signals correspond to the dichlorophenyl group of the ligand, indicating that this portion of the sensor does not experience conformational restrictions. However, the broadened VSD signals are a typical mark of lack of conformational freedom. This marked contrast between the behavior of VoLDeMo in organic and aqueous environment led us to formulate the following binding model which is depicted on the right. In the bulk solution, the lipophilic VSD is encapsulated by the dextran polymer and thus shielded from the surrounding aqueous environment. Only upon ligand binding, the VSD will be able to partition into the lipid bilayer.

\section{Slide 6}

We then probed the targeting of VoLDeMo probes in HEK cell cultures. The experimental setup is shown on the top. We used HEK cells stably transfected with the human dopamine transporter (or hDAT) as the protein target and a control HEK cell line transfected with an empty (null) vector. Three experimental conditions were probed. On the left, the VoLDeMo probe is incubated with hDAT-HEK cells, where association of the probe with hDAT is expected. The middle condition also involved hDAT-HEK cells but with pre- and co-incubation with the DAT blocker nomifensine, which occupies the target protein and thus should prevent VoLDeMo binding to the cell surface. Finally, in the experiment on the right, VoLDeMo is also not expected to associate with the null-HEK cells which lack hDAT. Indeed, clear membrane localized staining was observed upon treatment of hDAT-HEK cells with the dsRVF5-VoLDeMo probe as shown in the bottom figure, and this labeling was greatly diminished in both negative controls. The graph on the right shows quantification of this labeling selectivity.

\section{Slide 7}

After showing that VoLDeMo probes selectively target hDAT expressing cells, we aimed to verify whether the probe retains voltage sensitivity of the parent untethered probe. We labeled hDATHEK cells with dsRVF5-VoLDeMo and subjected the cells to whole cell patch clamp. We then controlled the membrane voltage of the cells in voltage clamp mode from $-100 \mathrm{mV}$ to $+80 \mathrm{mV}$ in 30-mV increments and recorded 1-photon excitation fluorescence. As shown in the bottom left figure, we observed clear increase in fluorescence with increasing membrane voltage. We then plotted the per-cent change of fluorescence over basal fluorescence (or $\Delta F / F$ ) versus membrane voltage (shown on the right), which gave a linear dependence with a slope of $13 \% \Delta F / F$ per $100 \mathrm{mV}$. Targeted dsRVF5-VoLDeMo thus exhibited good voltage sensitivity in cultured cells. 


\section{Slide 8}

We next proceeded into a way more challenging experimental system - acutely prepared mouse brain slices. We were interested specifically in the nigrostriatal dopaminergic system. As you can see in the cartoon on the top, this pathway consists of dopaminergic neurons which have cell bodies in the substantia nigra pars compacta (denoted as SNc) and project long axons through half of the brain to the dorsal striatum (denoted as dSTR) where they heavily arborize into a dense axonal network. We prepared striatal brain slices and incubated them with 100 nM dsRVF5VoLDeMo. As shown in the bottom left image, we observed a string-like fluorescence pattern consistent with the arborizing dopaminergic axons. As two negative controls, we either blocked DAT with nomifensine (shown in the middle) or carried out the labeling experiment with the naked voltage sensor dsRVF5. Only diffuse background staining was observed under both of these conditions. Such a high selectivity of labeling of natively expressed proteins is unprecedented for lipophilic VSDs in the context of brain tissue.

\section{Slide 9}

We then aimed to show that VoLDeMo probes can be used for recording voltage activity in brain tissue. Such recording with VoLDeMo probes turned out to be very challenging due to the low physiological expression levels of natively expressed DAT (or other similar targets). Therefore, we are operating with a photon budget perhaps two to three-orders of magnitude lower than that with overexpressed GEVI sensors.

The description of the experiment starts with the figure on the top right. We labeled striatal slices with dsRVF5-VoLDeMo and placed a bipolar electrode close to the region of interest and stimulated the slice delivering 25 pulses at $50 \mathrm{~Hz}$ while recording $2 \mathrm{P}$-excitation fluorescence by spiral scanning. A single illustrative frame of this spiral-scanning is shown on the top left. We chose this slow 2P-excitation approach to compensate for the low photon budget we were operating with. We also had to sacrifice high optical resolution to be able to scan at least at a $30 \mathrm{~Hz}$ frame rate. To account for signal changes that occur independent of the propagation of action potentials, we repeated the same experiment in the presence of the sodium channel blocker tetrodotoxin (TTX). The graph on the bottom left represents this recording as the averaged signal in the absence of TTX with the averaged signal in the presence of TTX subtracted as background. We observed a clear increase in fluorescence during the stimulation period, confirming that we can observe electrically induced activity of striatal dopaminergic axons. This is also quantified in the graph on the right which represents the averaged signal during the halfsecond stimulation period compared to the averaged signal in the half-second before stimulation started. Although the signal is low and signal averaging of many repeats was necessary to obtain useful signal, we demonstrate here the very first optical voltage recording from a specific cell type in the tissue of a wild-type animal without the use of any genetic manipulation. Please note that during the stimulation period, we observed a "dip" in the signal which may be genuine and may 
represent a circuitry effect with a period of relative inactivity. This effect is currently under further investigation in our laboratory.

\section{Slide 10}

To show that the design of our VoLDe platform is universal, we also modified this platform to enable covalent targeting of AMPA-type glutamate receptors (or AMPAR). We utilized the "covalent AMPAR modification" (or CAM) strategy developed by the group of Dr. Itaru Hamachi in Kyoto. The CAM strategy adopted to our system is explained in the figure on the top. The polymer carrier (blue) carries the voltage sensor (pink) and an AMPAR-selective ligand with an acyl imidazole linker (green). Upon binding to the receptor, a nearby lysine residue (red) attacks the acyl imidazole, covalently binds to the probe, and releases the ligand. Using a similar approach as used for the synthesis of VoLDeMo probes, we synthesized a dextran-based probe with the structure shown below. The dextran (blue) carries the dsRVF5 voltage sensor (purple) and an AMPAR-selective PFQX ligand (green) with the acyl imidazole cleavable linker (red). We termed this probe VoLDeCAM, as a fusion of our VoLDe class of probes with Hamachi's CAM targeting strategy. Notably, the dextran - $\mathrm{OH}$ groups were compatible with the cleavable acyl imidazole group.

\section{Slide 11}

We investigated VoLDeCAM labeling in acute mouse cortical slices. We focused on the somatosensory cortex which is highlighted in red in the graphic on the top. I would like to note that unlike the dopamine transporter, the AMPA receptor is a major receptor for the excitatory neurotransmitter glutamate and is therefore widely expressed in many neuronal subtypes. However, it is still a very interesting target due its postsynaptic localization. As you can see in the bottom left image, cortical slices incubated with dsRVF5-VoLDeCAM produced a dense punctate labeling pattern as recorded by 2-photon excitation fluorescence, which is consistent with the distribution of AMPA-receptors. Only a weak diffuse labeling pattern was observed in two negative controls, which involved blockage of the AMPA-receptor with the inhibitor NBQX or using a truncated control probe which lacked the ligand group. These results demonstrate the modularity of our VoLDe targeting platform in terms of different ligands and targeting strategies.

\section{Slide 12}

We tested the voltage sensitivity of dsRVF5-VoLDeCAM in cortical brain slices by 2-photon excitation fluorescence after electric stimulation. The experiment was carried out analogously to what I have shown for the VoLDeMo probe on slide 9. As shown on the top, we stimulated the slice using a bipolar electrode delivering 25 pulses at $50 \mathrm{~Hz}$ while recording 2-photon images with 
spiral scanning. After signal averaging from multiple trials and subtraction of background recorded in the presence of TTX, we obtained the graph on the bottom left which shows clear increase in fluorescence during the stimulation period. The average voltage increase during stimulation versus before stimulation is quantified in the bar graph on the right. An average $\Delta F / F$ of $0.28 \%$ was recorded which is higher than that recorded in striatal slices (where we recorded $0.12 \% \Delta F / F)$. We attribute this to a higher number of collected photons from a denser labeling of AMPA-receptors by VoLDeCAM compared to dopaminergic axons by VoLDeMo.

\section{Slide 13}

Finally, we sought to use dsRVF5-VoLDeCAM to record depolarization events in individual voltageclamped neurons in brain tissue. This experiment was not possible with the VoLDeMo probes as these do not label cell bodies well, due to the very low expression levels of these transporters on cell bodies compared to axons and dendrites. In contrast, VoLDeCAM produced a robust label of AMPA-receptors on all neuronal segments, including cell bodies. As shown on the top right, we labeled cortical slices with dsRVF5-VoLDeCAM and subjected a layer $V$ neuron to whole-cell patchclamp. In the voltage-clamp mode, we then induced five, $100-\mathrm{mV}$, half second depolarization steps from $-60 \mathrm{mV}$ to $+40 \mathrm{mV}$ as shown in the diagram on the top left. As a control, we kept the neuron at a constant potential of $-60 \mathrm{mV}$. We imaged the neurons with 1-photon excitation fluorescence during the course of these voltage steps. After averaging the voltage steps from multiple trials, we obtained the graph on the bottom left, where the blue trace represents the $100-\mathrm{mV}$ depolarization step while the gray trace represents the non-stimulated control. A clear increase in membrane potential during the depolarization period (highlighted in yellow and a red bar) was observed, while no significant increase in fluorescence was observed during the same time period when the membrane voltage was kept constant. The quantification is shown in the bar graphs on the right. The middle graph corresponds to the blue trace in the left graph and shows the averaged fluorescence during the $100-\mathrm{mV}$ stimulation (red) compared to resting potential (green). The graph on the right, which corresponds to the grey trace in the left graph, represents the constant potential control. Even in this experiment, averaging of multiple trials was required to extract useful signal. It is worth noting, though, that due to the dense VoLDeCAM labeling pattern, photons were collected not only from the cell body of interest but also from the surrounding neuropil which contributed only to the background signal. Therefore, the real magnitude of the signal is somewhat underestimated.

\section{Slide 14}

To summarize, we have developed a targeting platform for the delivery of highly lipophilic voltage sensitive dyes to specific cell types in brain tissue without the use of genetic manipulation. To achieve this, we created a delivery vehicle by combining the polysaccharide dextran with a highaffinity small molecule ligand and the lipophilic payload. Our VoLDeMo and VoLDeCAM sensors 
enabled the first exploration of optical voltage imaging with probes targeted to native membrane proteins at their physiological expression levels. We project that the modularity of this platform will allow for delivery of other payloads such as lipophilic drugs or actuators in the brain tissue.

\section{5}

This work was carried out as a collaboration between the groups of Professors Dalibor Sames and David Sulzer at Columbia. I would like to acknowledge Jihang Wang who has carried out a majority of the work in acute brain slices. I would like to thank Dr. Yuki Bando from the Yuste group at Columbia Kavli Institute for his help with patch clamp experiments in cell cultures. I also thank other people, who contributed to this work, including Dr. Se Joon Choi who carried out the electrophysiology experiments in brain slices, and all other group members highlighted in green. Special thanks belong to our funding sources, including the Mathers Foundation, the National Institutes of Health, and the Alfred Bader Foundation. If you are interested in details of our work, please check out our paper which will be coming out soon in JACS. Thank you for your attention. 